\title{
Intravascular ultrasound catheter for transesophageal echocardiography in congenital heart surgery -A case report-
}

\author{
Hye-Jin Kim ${ }^{1}$, Jae-Yun Kim ${ }^{1}$, Seung-Hoon Baek ${ }^{1,2}$, and Hae-Kyu Kim ${ }^{1,2}$ \\ ${ }^{1}$ Department of Anesthesiology and Pain Medicine, Pusan National University School of Medicine, Yangsan, ${ }^{2}$ Medical Research \\ Institute, Pusan National University Hospital, Busan, Korea
}

Transesophageal echocardiography (TEE) has an important role during congenital heart surgery. TEE in small infants is associated with complications, including an inability to pass the TEE probe, esophageal trauma, airway compression, aortic compression, and interference with ventilation. Recently, a monoplane intravascular ultrasound catheter (IVUC) has been developed for intracardiac echocardiography. The efficacy of IVUC for transesophageal use has been shown in numerous animal studies and several human studies, but there have been few reports involving small infants using an IVUC probe. We examined 15 pediatric patients undergoing congenital cardiac surgery using an 8-Fr AcuNav IVUC probe. We checked the cardiac anatomy, cardiac function pre-operatively and de-aeration before weaning from CPB; the surgical repairs were evaluated post-operatively. Although the IVUC probe has limitations associated with the monoplane, we found the IVUC probe to be useful in small infants and safer than the TEE. (Korean J Anesthesiol 2010; 58: 480-484)

Key Words: Congenital heart surgery, Intravascular ultrasound catheter, Transesophageal echocardiography.

Transesophageal echocardiography (TEE) during cardiac surgery permits cardiac assessment and provides the surgeon with information about the adequacy of surgical repair immediately after the procedure. However, even a pediatric omniplane TEE probe is too large for use in small infants. Indeed, TEE is limited in infants weighing under $5 \mathrm{~kg}$ (Fig. 1). A pediatric omniplane TEE probe will not pass the esophagus in neonates or very small infants. Even though a TEE probe can pass the esophagus in small infants, the probe compresses the endotracheal tube and surrounding structures, such as the distal trachea, mainstem bronchi, heart, and aorta [1,2].

Recently, a monoplane intravascular ultrasound catheter (IVUC, AcuNav ${ }^{\mathrm{TM}}$; SIEMENS Medical, CA, USA) has been developed. The IVUC was originally used for intravascular or intracardiac echocardiography (ICE) during cardiac catheterization; the IVUC has been proposed in several

Received: November 20, 2009. Revised: December 1, 2009. Accepted: December 8, 2009.

Corresponding author: Seung-Hoon Baek, M.D., Department of Anesthesiology and Pain Medicine, School of Medicine, Pusan National University, Beomeo-ri, Mulgeum-eup, Yangsan 626-770, Korea. Tel: 82-55-360-2129, Fax: 82-55-360-2149, E-mail: anebsh@pusan.ac.kr This work was supported for two years by Pusan National University Research Grant.

(c) This is an open-access article distributed under the terms of the Creative Commons Attribution Non-Commercial License (http:// creativecommons.org/licenses/by-nc/3.0/), which permits unrestricted non-commercial use, distribution, and reproduction in any medium, provided the original work is properly cited. 
studies for use during TEE in small animals or infants $[3,4]$. The IVUC probe is available in an $8 \mathrm{Fr}$ size (2.5 mm; Fig. 1). This is a 64-element vector, phased array transducer, which is multifrequency $(5.5-10 \mathrm{MHz})$ with a maneuverable four-way head articulation to allow multiple angle imaging. There is a steering lock to maintain angulation of the catheter. The probe is capable of high-resolution two-dimensional and full Doppler imaging (pulsed, continuous wave, and tissue Doppler). The longitudinal plane provides a 90-degree sector image with tissue penetration of $2-12 \mathrm{~cm}$.

In this study, we had 15 cases for TEE using an IVUC probe in small infants under $5 \mathrm{~kg}$ and determined its efficacy and safety.

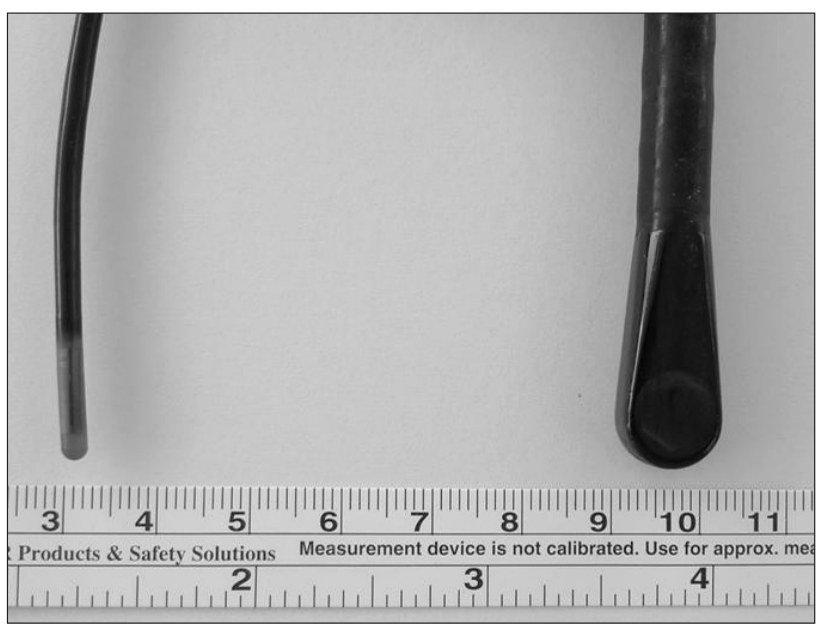

Fig. 1. Comparison between 8-Fr AcuNav IVUC (left) and pediatric omniplane TEE probe (right).

\section{Case Report}

We examined 15 consecutive neonates and infants who were undergoing congenital cardiac surgery between 1 May 2009 and 31 October 2009 using an 8-Fr AcuNav IVUC probe. Infants weighing 2-5 kg undergoing congenital heart surgery were studied. The characteristics of the patients, including the type of surgery, are presented in Table 1.

Anesthesia was induced with midazolam $(0.2 \mathrm{mg} / \mathrm{kg})$, fentanyl $(10 \mu \mathrm{g} / \mathrm{kg})$, and sevoflurane. For maintenance of anesthesia, all patients were administered sevoflurane and fentanyl with controlled ventilation. Electrocardiography, invasive arterial blood pressure, central venous pressure, pulse oximetry, temperature, capnography, and cerebral oximetry were monitored throughout the operation.

With the patient in the supine position, a lubricated IVUC probe was inserted through the mouth and passed into the esophagus without any complications. Because the probe does not have scale marks, the depth of placement was guided by echocardiography imaging.

TEE using an IVUC probe was able to detect atrial septal defects (ASDs; $\mathrm{n}=2$ ) or ventricular septal defects (VSDs; $\mathrm{n}=$ 11), and immediately after surgical repair (ASD, direct closure; VSD, patch closure), we confirmed if there was leakage from the direct or patch closure site and evaluated de-aeration in the cardiac chamber (Fig. 2).

After total correction for tetralogy of Fallot (TOF), it is important to examine the right ventricular outflow tract (RVOT), including blood flow to the pulmonary artery, pressure gradient, velocity, and VSD [5]. Fig. 3 shows how to check the post-

Table 1. Patient Characteristics and Surgical Procedures

\begin{tabular}{|c|c|c|c|c|}
\hline Patients & Age & Weight & Diagnosis & Surgical intervention \\
\hline 1 & $14 \mathrm{D}$ & $2.7 \mathrm{~kg}$ & $\begin{array}{l}\text { TOF, secundum ASD, } \\
\text { absent pulmonory valve syndrome }\end{array}$ & $\begin{array}{l}\text { Creation of a conduit between the right ventricle \& pulmonary artery, to- } \\
\text { tal correction of tetralogy of Fallot, primary closure of ASD }\end{array}$ \\
\hline 2 & $32 \mathrm{D}$ & $3.3 \mathrm{~kg}$ & VSD & VSD patch closure \\
\hline 3 & $8 \mathrm{D}$ & $3.0 \mathrm{~kg}$ & IAA, VSD, ASD & $\begin{array}{l}\text { Total correction (extended end-to-end anastomosis), } \\
\text { VSD patch closure, direct closure of ASD }\end{array}$ \\
\hline 4 & $18 \mathrm{D}$ & $3.1 \mathrm{~kg}$ & CoA, VSD & Total correction (extended end-to-end anastomosis), VSD patch closure \\
\hline 5 & $30 \mathrm{D}$ & $5.4 \mathrm{~kg}$ & CoA, VSD & Total correction (extended end-to-end anastomosis), VSD patch closure \\
\hline 6 & $8 \mathrm{D}$ & $3.2 \mathrm{~kg}$ & $\begin{array}{l}\text { Hemitruncus arteriosus (anoma- } \\
\text { lous origin of RPA: from aorta) }\end{array}$ & $\begin{array}{l}\text { Direct anastomosis between the RPA and MPA, } \\
\text { angioplasty of RPA orifice }\end{array}$ \\
\hline 7 & $7 \mathrm{D}$ & $2.9 \mathrm{~kg}$ & HLHS & Norwood procedure \\
\hline 8 & $6 \mathrm{D}$ & $4.0 \mathrm{~kg}$ & TGA & Arterial switch operation (Jatene procedure) \\
\hline 9 & $45 \mathrm{D}$ & $4.5 \mathrm{~kg}$ & VSD & VSD patch closure \\
\hline 10 & $3 \mathrm{M}$ & $4.8 \mathrm{~kg}$ & VSD & VSD patch closure \\
\hline 11 & $35 \mathrm{D}$ & $3.6 \mathrm{~kg}$ & VSD & VSD patch closure \\
\hline 12 & $28 \mathrm{D}$ & $2.5 \mathrm{~kg}$ & VSD & VSD patch closure \\
\hline 13 & $31 \mathrm{D}$ & $2.9 \mathrm{~kg}$ & VSD & VSD patch closure \\
\hline 14 & $71 \mathrm{D}$ & $4.9 \mathrm{~kg}$ & VSD & VSD patch closure \\
\hline 15 & $53 \mathrm{D}$ & $5.0 \mathrm{~kg}$ & VSD & VSD patch closure \\
\hline
\end{tabular}

D: day, M: month, TOF: tetralogy of Fallot, ASD: atrial septal defect, VSD: ventricular septal defect, IAA: interrupted aortic arch, CoA: coactation of aorta, HLHS: hypoplastic left heart syndrome, TGA: transposition of the great arteries, RPA: right pulmonary artery, MPA: main pulmonary artery. 

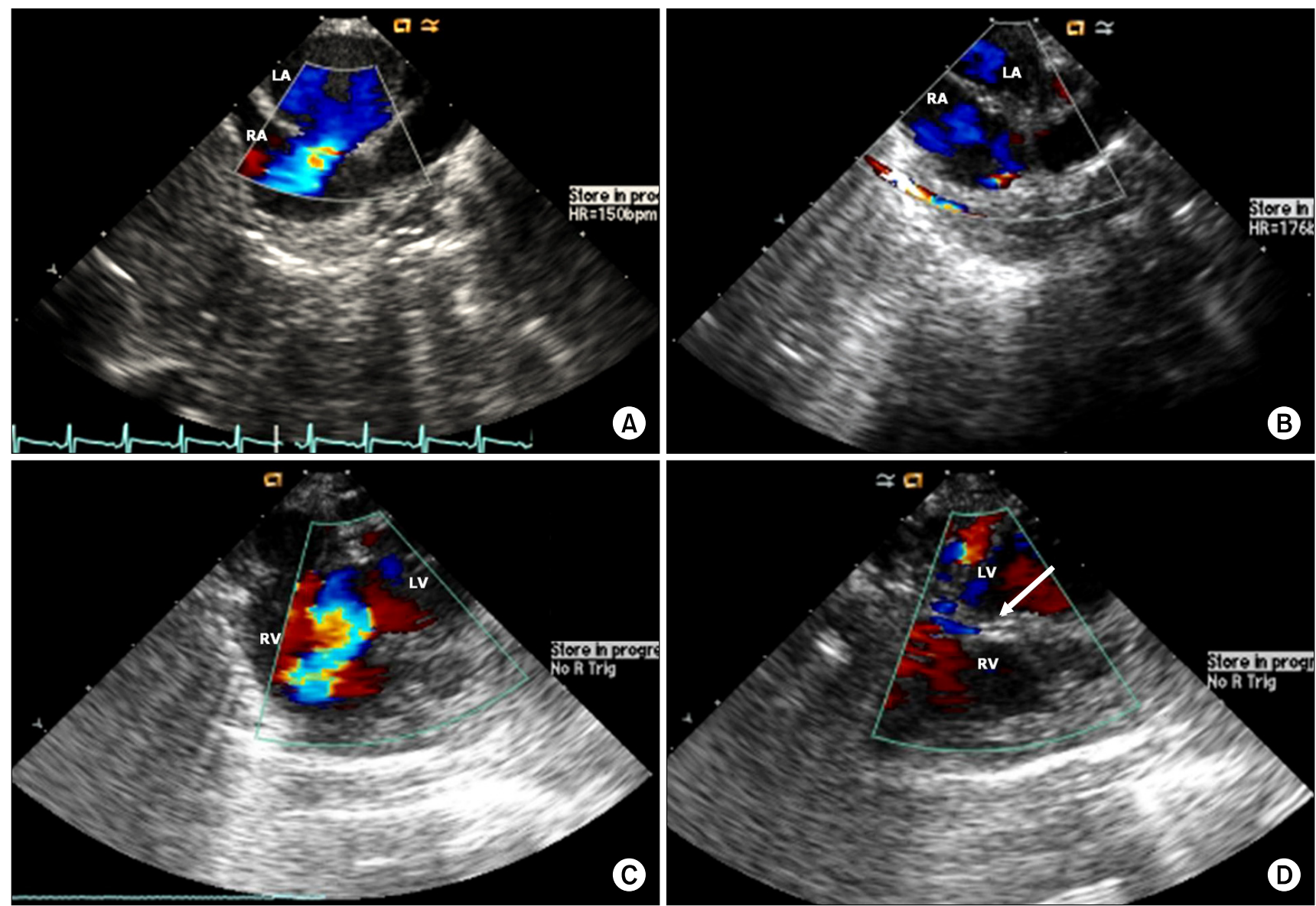

Fig. 2. (A) Atrial septal defect is seen from the mid-esophageal bicaval view with the 8-Fr IVUC AcuNav probe. Shunt flow through the defect is observed. (B) Atrial septal defect is repaired by primary closure. There is no leakage at the repaired site. (C) Ventricular septal defect before surgical repair. Left-to-right shunt is seen by the color Doppler. (D) Ventricular septal defect is closed by the patch. The arrow indicates an intact patch. There is no residual shunt flow through the patch. LA: left atrium, RA: right atrium, LV: left ventricle, RV: right ventricle.
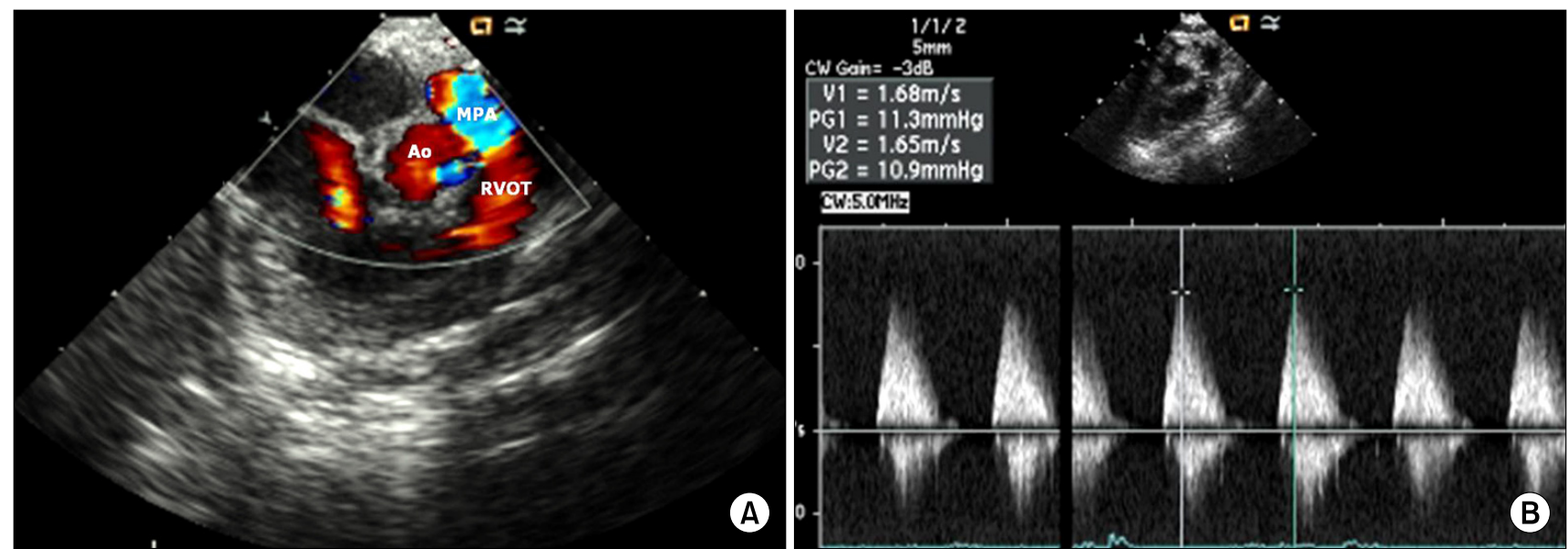

Fig. 3. (A) Right ventricular outflow tract after total correction for tetralogy of Fallot. There is no obstruction or stenosis through the right ventricular outflow tract. Ao: aorta, MPA: main pulmonary artery, RVOT: right ventricular outflow tract. (B) Measuring pulmonary artery pressure by the continuous wave Doppler. The pressure gradient and velocity through the pulmonic valve is checked within the normal range. 

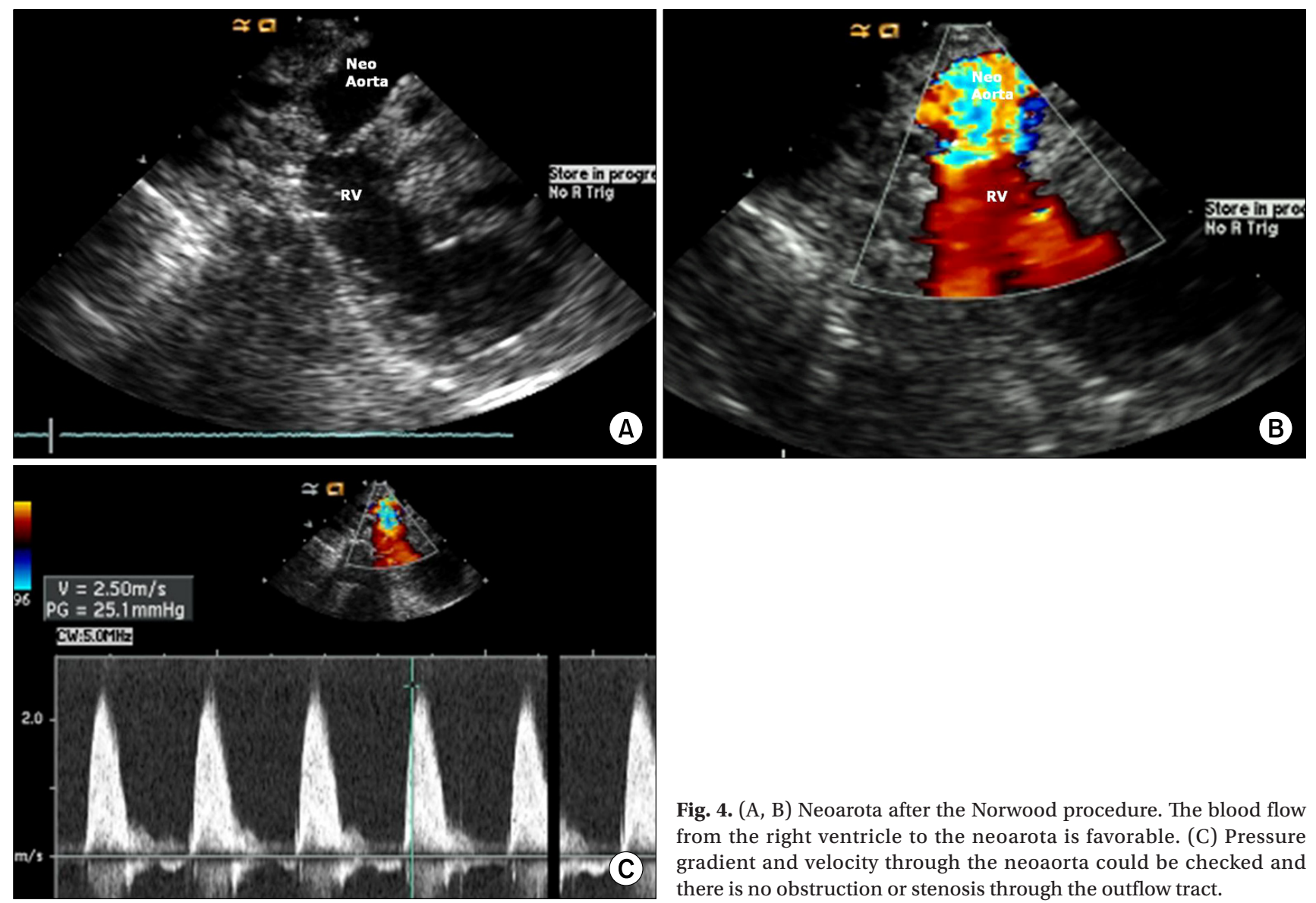

Fig. 4. (A, B) Neoarota after the Norwood procedure. The blood flow from the right ventricle to the neoarota is favorable. (C) Pressure gradient and velocity through the neoaorta could be checked and there is no obstruction or stenosis through the outflow tract.

operative pulmonary artery pressure gradient and velocity using an ICE probe. In this patient, we clearly evaluated the RVOT, leakage of the patch closure site, the main pulmonary artery, and the pressure gradient between the pulmonary valves.

During the period of study, there was a patient with hypoplastic left heart syndrome (HLHS) who underwent a Norwood procedure. We evaluated the neoaorta (conduit from the right ventricle to the pulmonary artery) after completion of the procedure [6]; blood flow through the neoaorta was measured, as shown in Fig. 4.

There were no patients with complications related to introduction of the probe or its use during the surgical procedure.

\section{Discussion}

Heart surgery for congenital heart disease in very small infants is often carried out, but it has been difficult to use conventional pediatric omniplane TEE probes in such patients. As a result, we could not previously assess the information about the anatomy, surgical repair, and heart function in patients weighing under $5 \mathrm{~kg}$. Recently, the IVUC probe has been applied to animals and neonates as a TEE, proving it useful and safe. We also experienced the feasibility and usefulness of the IVUC probe based on 15 cases in our hospital.

The AcuNav IVUC probe is reasonable in small infants weighing under $5 \mathrm{~kg}$, but it has several limitations. Because the AcuNav IVUC probe has monoplane features, it is difficult to fully appreciate the three-dimensional nature of the complex cardiac anatomy and the IVUC probe requires significant manipulation, which can be challenging in small patients. In cases involving simple VSD, ASD, and TOF, the defects and the surgical repairs were easily demonstrated. However, more complex congenital diseases, like anomalous origins of the right pulmonary artery (RPA; Table 1), could not be easily assessed.

The probe utilized in this study is not steerable and has only one imaging plane. Even though the probe has a four-way head articulation, it was difficult to obtain standard long-axis twoor four-chamber images of the heart on a case-by-case, and it was not easy to gain transgastric images comparable to TEE with a conventional probe. Moreover, the current study did not include patients with oropharyngeal anomalies in whom the conventional TEE probe could not be passed and for which the 
IVUC probe may be a useful tool.

Mart et al. [7] reported successful TEE evaluation in a $1.4 \mathrm{~kg}$ infant with complex congenital heart disease. They dilated the esophagus prior to inserting the TEE probe. Although intraoperative TEE can be safely performed, even in small infants, it frequently causes mild mucosal injuries to the esophagus [8]. The TEE probe can cause vascular compression or ventilatory compromise $[1,2]$. We suggest that an IVUC for TEE in small infants eliminates any complications in patients previously thought to be too small for intra-operative TEE.

The IVUC probe is designed for single use when it is applicated to intravascular access. IVUC costs about three million won per probe, it is very expensive for single use. But in the case of transesophageal use, it can be reusable like other conventional TEE probe. Considering that IVUC has the term of validity ( 2 months), we could use 2 probes for 15 cases with sterilization. We obtained good image quality at every case and its cost per patient was similar as conventional TEE.

Finally, as though it is difficult to get obvious images in some cases, anyone who is familiar with conventional TEE can use this IVUC with only several attempts.

In conclusion, although the IVUC probe is disadvantageous based on its monoplane features and not being steerable, we found the IVUC probe useful for TEE in small infants and safe without any complications, such as hemodynamic instability, difficulty in ventilation, or esophageal injury.

\section{References}

1. Andropoulos DB, Ayres NA, Stayer SA, Bent ST, Campos CJ, Fraser $\mathrm{CD}$. The effect of transesophageal echocardiography on ventilation in small infants undergoing cardiac surgery. Anesth Analg 2000; 90: 47-9.

2. Andropoulos DB, Stayer SA, Bent ST, Campos CJ, Fraser CD. The effects of transesophageal echocardiography on hemodynamic variables in small infants undergoing cardiac surgery. J Cardiothorac Vasc Anesth 2000; 14: 133-5.

3. Drinker LR, Camitta MG, Herlong JR, Miller S, Lodge AJ, Jaggers J, et al. Use of the monoplane intracardiac imaging probe in high-risk infants during congenital heart surgery. Echocardiography 2008; 25: 999-1003.

4. Lu L, Ko E, Schwartz GG, Chou TM. Transesophageal echocardiography in rats using an intravascular ultrasound catheter. Am J Physiol 1997; 273: H2078-82.

5. Pongiglione G, Freedom RM, Cook D, Rowe RD. Mechanism of acquired right ventricular outflow tract obstruction in patients with ventricular septal defect: an angiocardiographic study. Am J Cardiol 1982; 50: 776-80.

6. Cardis BM, Fyfe DA, Ketchum D, Mahle WT. Echocardiographic features and complications of the modified norwood operation using the right ventricle to pulmonary artery conduit. J Am Soc Echocardiogr 2005; 18: 660-5.

7. Mart CR, Fehr DM, Myers JL, Rosen KL. Intraoperative transesophageal echocardiography in a 1.4-kg infant with complex congenital heart disease. Pediatr Cardiol 2003; 24: 84-5.

8. Greene MA, Alexander JA, Knauf DG, Talbert J, Langham M, Kays D, et al. Endoscopic evaluation of the esophagus in infants and children immediately following intraoperative use of transesophageal echocardiography. Chest 1999; 116: 1247-50. 